\title{
Radiological and clinical findings of osseous peripheral primitive neuroectodermal tumors
}

\author{
CHAO WANG, BIN LI, XIN-FENG YU, MIN XUAN, QUAN-QUAN GU, WEI QIAN, \\ TIAN-TIAN QIU, ZHU-JING SHEN and MIN-MING ZHANG
}

\begin{abstract}
Department of Radiology, The Second Affiliated Hospital of Zhejiang University School of Medicine, Hangzhou, Zhejiang 310009, P.R. China
\end{abstract}

Received August 5, 2014; Accepted May 7, 2015

DOI: $10.3892 / \mathrm{ol} .2015 .3233$

\begin{abstract}
Peripheral primitive neuroectodermal tumor (pPNET) is a rare and highly malignant undifferentiated type of tumor. The aim of the present study was to analyze the computed tomography $(\mathrm{CT})$, magnetic resonance imaging (MRI) and clinical findings of osseous pPNET. The present study retrospectively analyzed the clinical data and CT findings from 15 patients with osseous pPNET; the MRI findings from 11 of these 15 patients were confirmed by histopathological examination. The 15 patients included 9 men and 6 women. The mean patient age was 29 years (range, 16-64 years) and 11 cases were aged $<30$ years. A CT scan was performed in 15 cases and the findings included a lytic lesion (13 cases), a lytic lesion with irregular sclerosis and dilation (2 cases), a soft tissue mass (15 cases), calcification ( 2 cases) and periosteal reaction (5 cases). A total of 9 cases of soft tissue mass were heterogeneous, with different sizes of lower-density necrotic areas. An enhanced MRI scan was performed in 11 cases. On T1-weighted images (WI), the soft tissue mass was isointense ( 8 cases) and marginally hyperintense ( 3 cases). On T2WI, aggressive soft tissue masses were heterogeneous iso- or hyperintense (11 cases). On contrast-enhanced T1WI, marked heterogeneous enhancement was present in 10 cases and intermediate heterogeneous enhancement in 1 case. The results indicated that osseous pPNET mainly affects male adolescents and young adults. The CT findings of osseous pPNET were destructive lesions with a soft tissue mass and, occasionally, with periosteal reaction. The tumor was often isodense, with patchy hypodense areas. Tumor calcification was uncommon. The MRI findings were those of an aggressive soft tissue mass exhibiting isointensity on T1WI and iso- or hyperintensity on T2WI, with marked heterogeneous enhancement. Although
\end{abstract}

Correspondence to: Professor Min-Ming Zhang, Department of Radiology, The Second Affiliated Hospital of Zhejiang University School of Medicine, 88 Jiefang Road, Hangzhou, Zhejiang 310009, P.R. China

E-mail: zhangminming@zju.edu.cn

Key words: bone neoplasm, primitive neuroectodermal neoplasm, computed tomography, magnetic resonance imaging the imaging characteristics of pPNETs may be non-specific, CT and MRI may be useful in delineating the extent of the tumor, identifying distant metastases, predicting resectability and monitoring treatment.

\section{Introduction}

Primitive neuroectodermal tumors (PNETs) are rare, highly malignant neoplasms consisting of small round cells of neural crest origin (1). PNETs may be further subdivided into central and peripheral PNETs (pPNETs), which arise outside the central and sympathetic nervous system. pPNETs often occur in the soft tissues of thoracopulmonary region (Askin tumor), retroperitoneum and abdomen, and, more rarely, in the bones (1-3). There is no consensus with regard to the guidelines for the treatment of pPNET at present, due to its rare occurrence. As PNET exhibits similarities to Ewing sarcoma, surgical resection followed by adjuvant radiotherapy, as well as multiagent chemotherapy if possible, is considered necessary to improve patient survival. There have been few studies published exclusively on the osseous pPNET (3), particularly regarding its radiological and clinical features. Therefore, the present study aimed to document the radiological and clinical features of osseous pPNET by conducting a retrospective radiological and clinical review of 15 patients with surgically or bioptically confirmed osseous pPNET.

\section{Materials and methods}

Patients. The database of the Second Affiliated Hospital of Zhejiang University School of Medicine (Hangzhou, China) was searched for the records of patients with pPNET who were treated between January, 2011 and January, 2014. A total of 17 patients with pPNET were identified; 2 patients with extraosseous pPNET were excluded and 15 patients with osseous pPNET were finally included in the study. An Institutional Review Board exemption and a waiver for the requirement of written informed consent were obtained to perform this retrospective study.

Imaging. All 15 patients had undergone computed tomography (CT) and 11 had also undergone magnetic resonance imaging (MRI).CT imaging was performed using a Somatom Sensation 

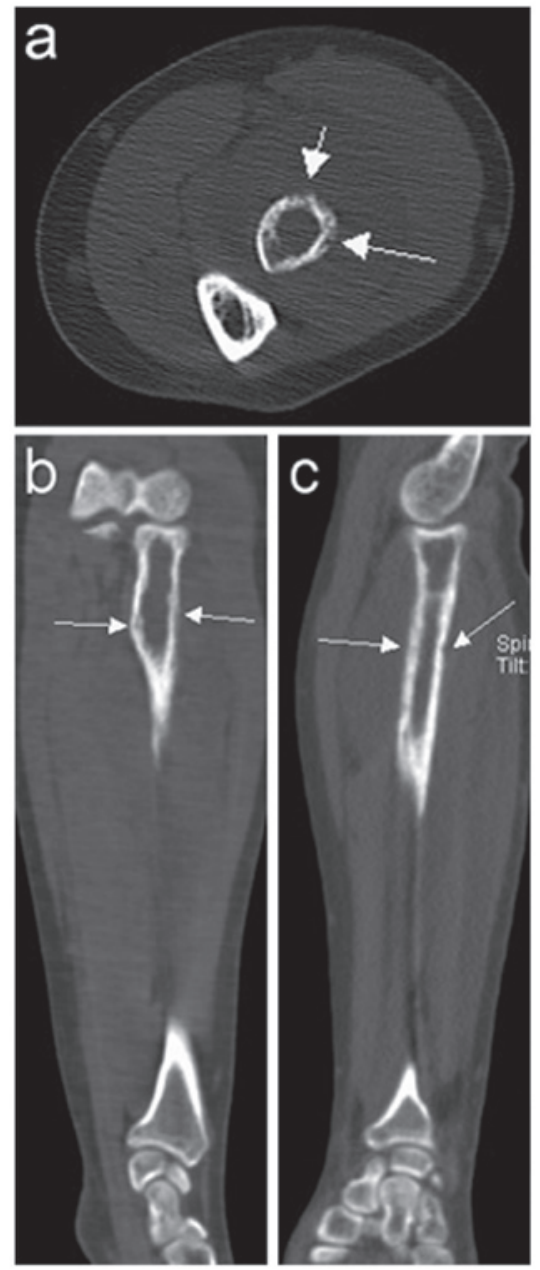

Figure 1. Patient 1. Lytic bone lesions in the left upper radius (arrows) visble on (a) axial, (b) coronal and (c) sagittal images.

16 helical scanner (Siemens Healthcare, Erlangen, Germany). The scanning parameters were as follows: 5 -mm slice thickness reconstructions for viewing, 1-mm slice thickness reconstructions for post-processing, B40s medium kernel, 20-cm field of view, $120 \mathrm{kV}$ voltage, 200-300 mA current and $512 \times 512$ matrix. MRI was performed using a 3.0T GE Signa MRI scanner (GE Healthcare, Little Chalfont, UK). The scan parameters were as follows: T1-weighted fast spin echo (FSE) sequence [repetition time/echo time (TR/TE), 500/10 msec; slice thickness, $5.0 \mathrm{~mm}$; field of view, $380-520 \mathrm{~mm}$; and matrix scan, 256x 256] and T2-weighted turbo-spin echo sequence (TR/TE, 3,000/75 msec; slice thickness, $3.0 \mathrm{~mm}$; field of view, 300-380 mm; and matrix scan, 256x256). An intravenous dose of 0.1-0.2 mmol $/ \mathrm{kg}$ of contrast agent (Gadolinium-diethylene triamine pentaacetic acid; Magnevist ${ }^{\circledR}$; Bayer Schering Pharma AG, Berlin, Germany) was administered to the patients undergoing contrast-enhanced MRI. A total of 7 patients underwent chemotherapy $(n=3)$ or chemoradiotherapy $(n=4)$. For 2 of those cases MRI data were also available following chemotherapy.

\section{Results}

Clinical data. The study group included 9 men and 6 women with a mean age of 29 years (range, 16-64 years). The tumors were located in the maxilla (1 case), mandible (1 case), humerus
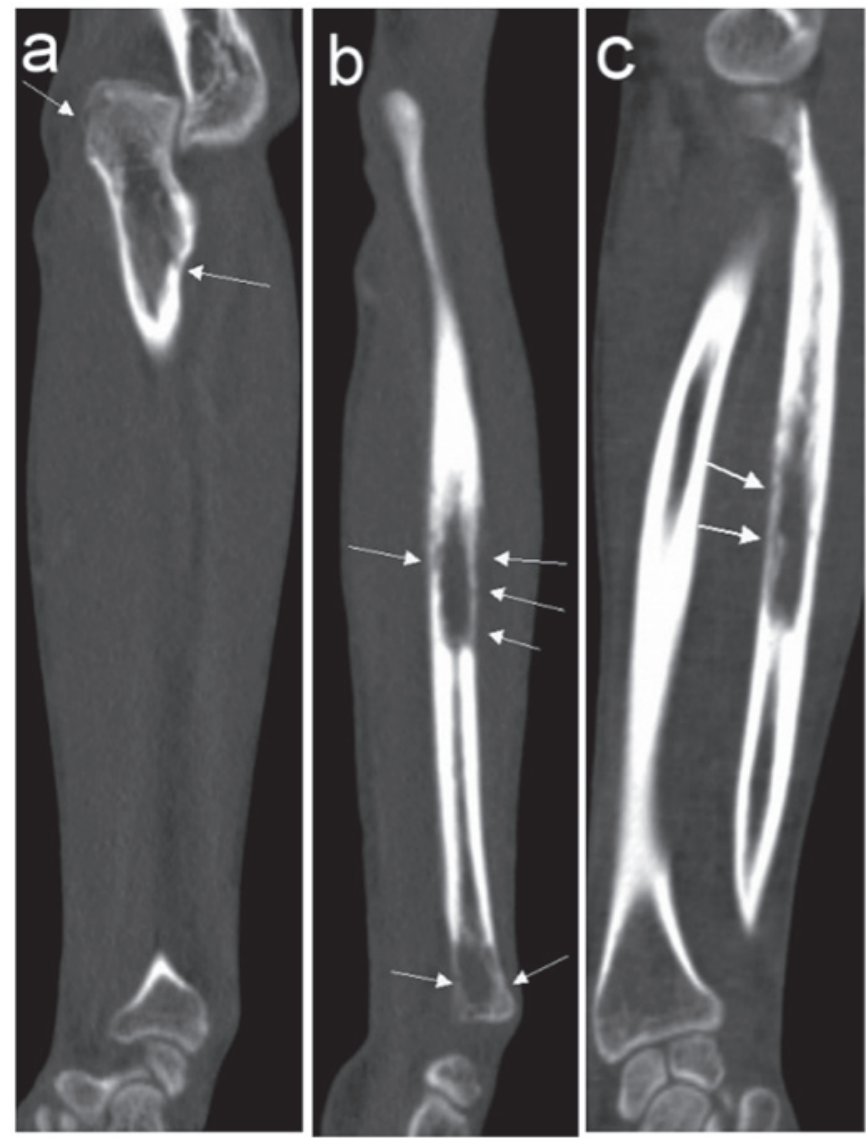

Figure 2. Patient 13. Lytic bone lesions of the left (a) upper, (b) middle, and (c) lower ulna (arrows).

( 1 case), radius ( 1 case), fibula (1 case), femur ( 1 case), scapula ( 1 case), ilium (1 case), cervical vertebrae (1 case), lumbar vertebrae (1 case), clavicle ( 2 cases), tibia ( 2 cases) and ulna ( 2 cases). One tumor was located in the tibia and fibula in the same patient (Table I). A total of 15 patients presented with varying degrees of pain; of these, 11 patients presented with local edema and a progressive, growing mass.

CT findings. The radiological findings from the 15 cases are summarized in Table I. A CT scan was performed in all 15 cases. A total of 13 cases exhibited lytic bone lesions (Figs. 1 and 2) and the remaining 2 cases exhibited lytic bone lesions with bone expansion (Figs. 3 and 4). All 15 cases exhibited surrounding soft tissue mass formation; of the soft tissue masses, 9 cases were heterogeneous, with different sizes of lower-density necrotic areas (Fig. 5). The CT value of solid sections of the tumors was 40-65 HU. All 15 cases exhibited a relatively limited extent of bone cortical destruction, with surrounding soft tissue mass formation (Figs. 1 and 2). Two cases of soft tissue mass exhibited calcification (Figs. 4 and 5) and 5 cases exhibited periosteal reaction, including clear sunburst-like periosteal reactions in 3 cases (Figs. 6 and 7). Two cases of vertebral pPNET exhibited a soft tissue mass convex to the spinal canal, causing spinal cord compression (Fig. 8).

MRI findings. Contrast medium-enhanced MRI scanning was performed in 11 cases. All 11 cases exhibited osseous signal abnormalities and the confines of the lesions were wider 


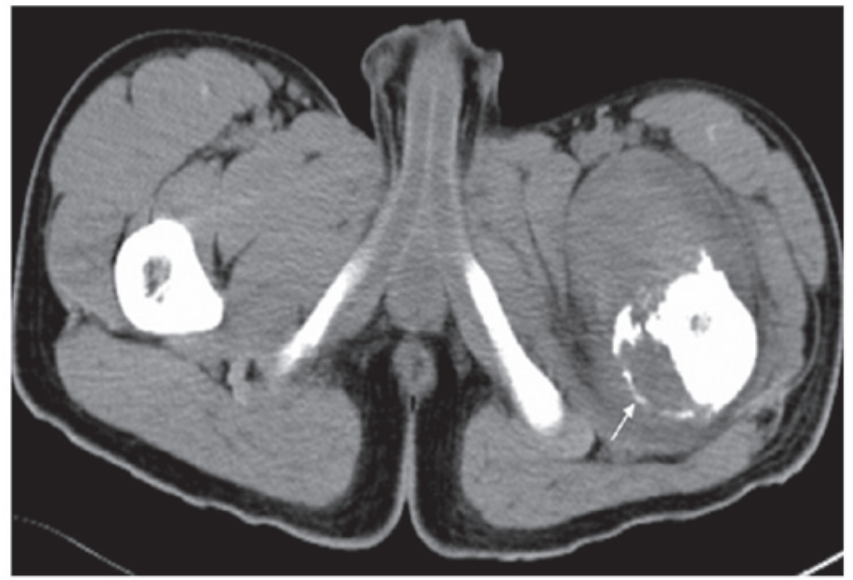

Figure 3. Patient 7. Lytic bone lesion of the left femur, with irregular sclerosis (arrow) and bone expansion.

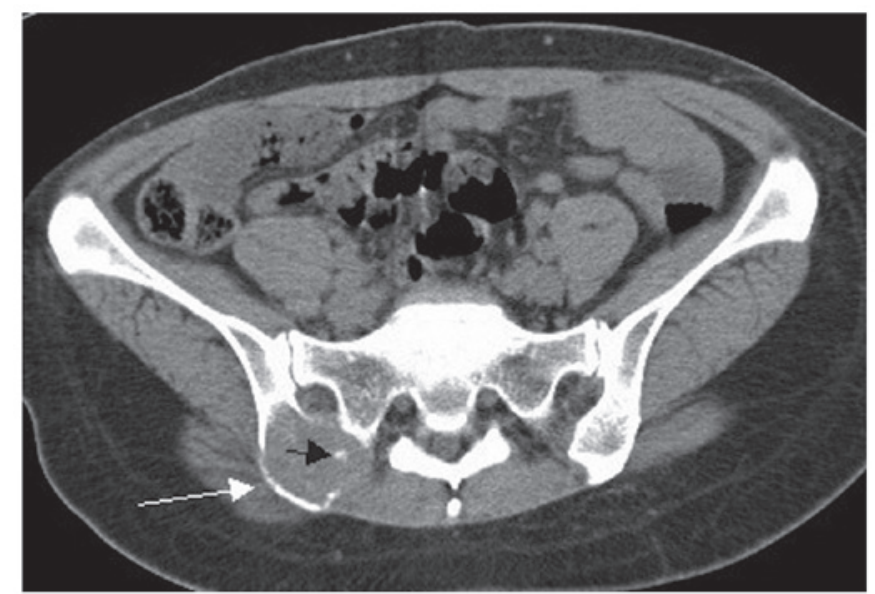

Figure 4. Patient 10. Lytic bone lesion of the right ilium, with irregular sclerosis and bone expansion (white arrow), and calcification (black arrow).

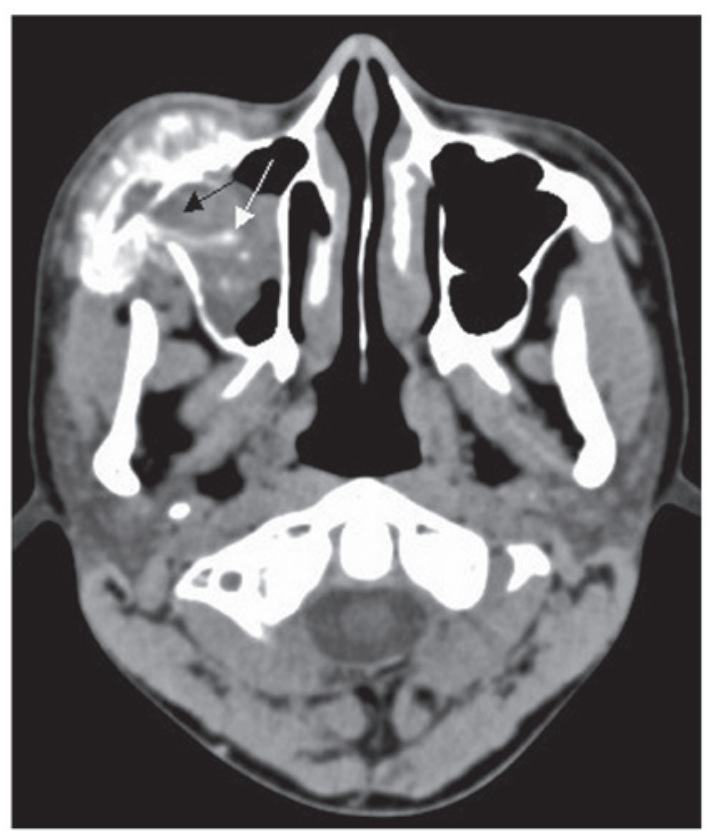

Figure 5. Patient 3. Soft tissue mass in the right maxillary sinus, exhibiting heterogeneity with different sizes of lower-density necrotic areas (black arrow) and calcification (white arrow).

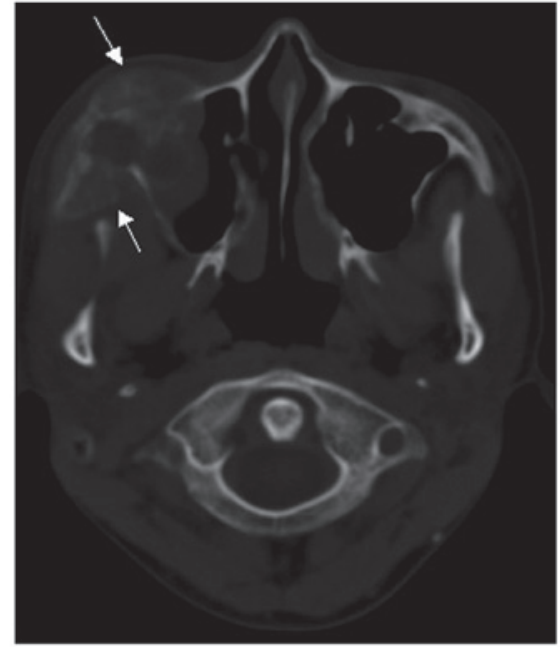

Figure 6. Patient 3. Right maxillofacial tumor (bone windows) with cortical destruction of the wall of the right maxillary sinus and sunburst-like periosteal reactions (arrows).

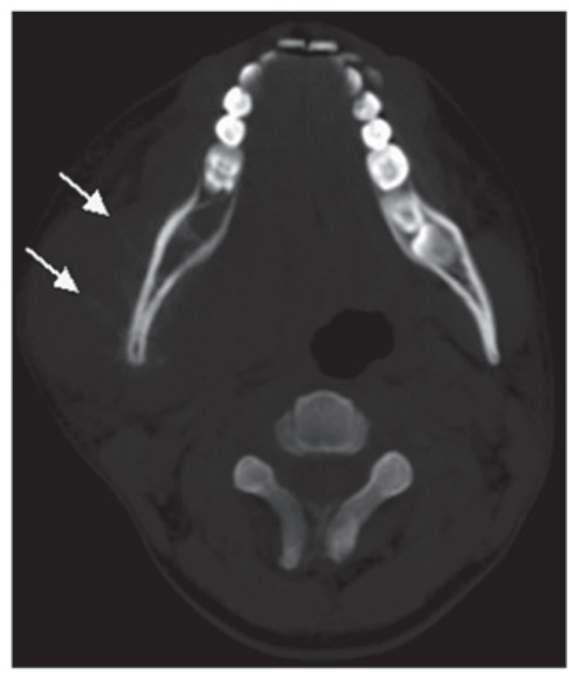

Figure 7. Patient 9. The bone windows show cortical destruction and sunburst-like periosteal reactions of the right mandible (arrows).

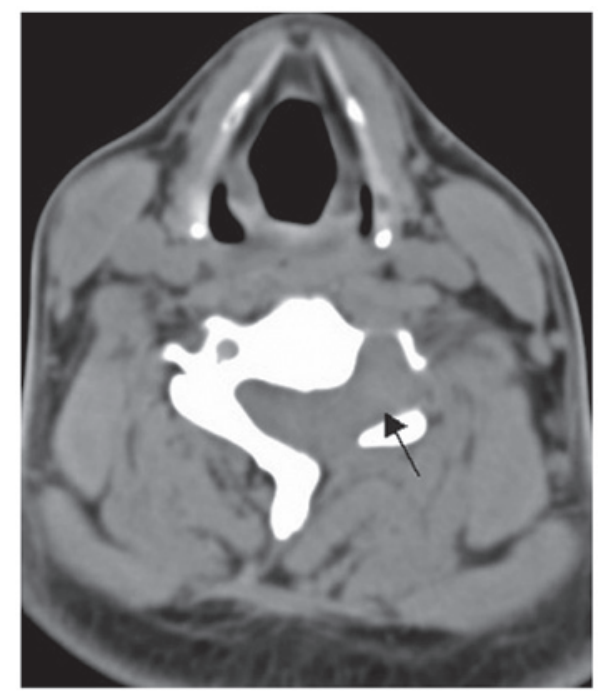

Figure 8. Patient 15. Left cervical intervertebral foramen with a soft tissue mass convex to the spinal canal, causing spinal cord compression (arrow). 

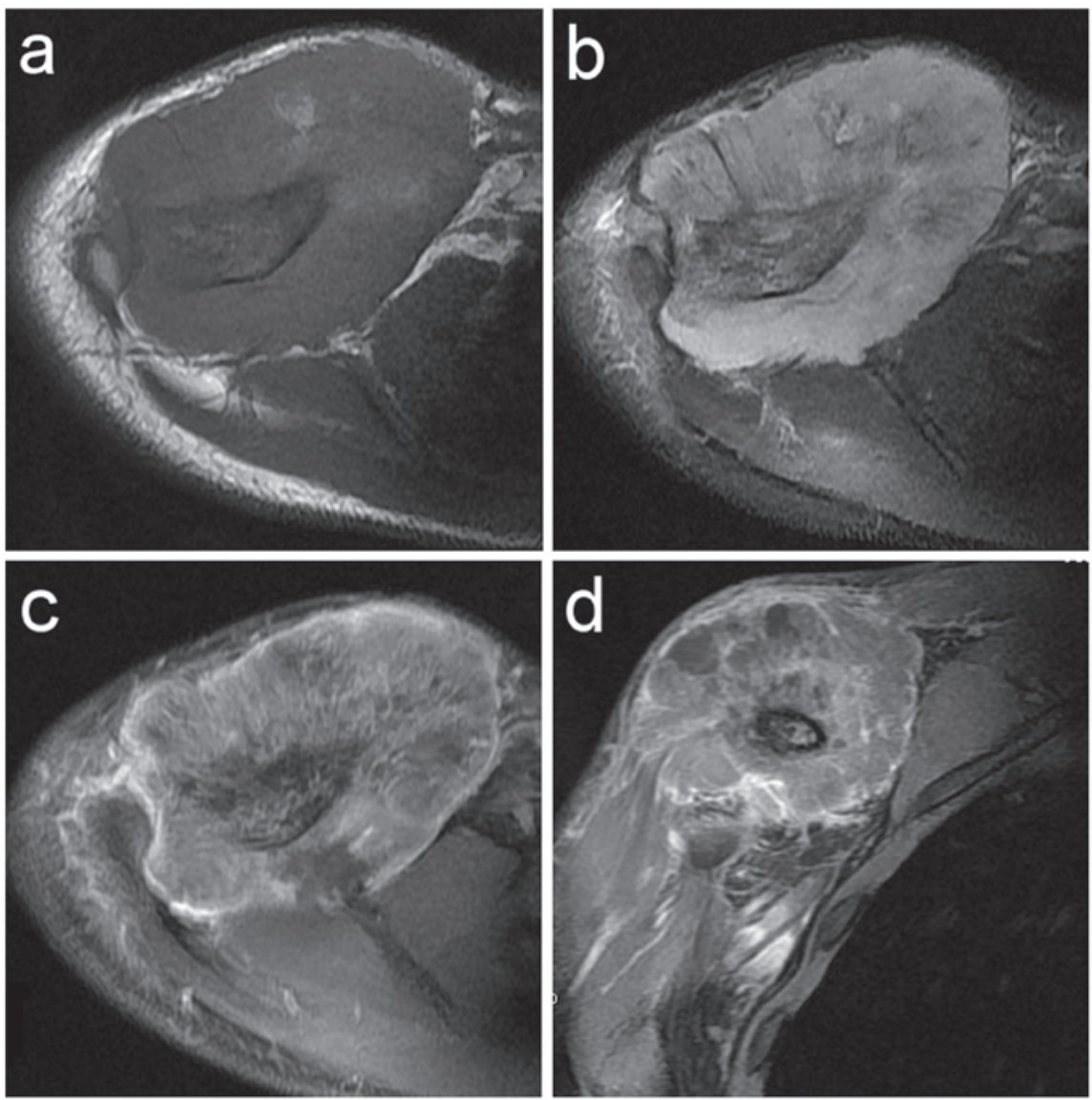

Figure 9. Patient 12. (a) Soft tissue mass of the right clavicle exhibiting isointensity on T1WI; (b) aggressive soft tissue mass with heterogeneous iso- or hyperintensity detected around the right clavicle on T2WI; and (c and d) marked heterogeneous enhancement on contrast-enhanced T1WI. WI, weighted images.

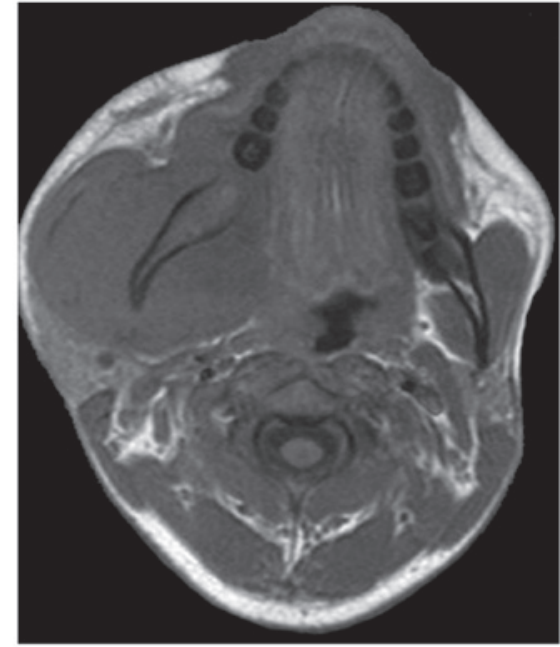

Figure 10. Patient 9. Soft tissue mass of the right mandible exhibiting isointensity on T1-weighted images.

compared with those identified on CT. All 11 cases exhibited a surrounding soft tissue mass. On T1-weighted images (WI), a soft tissue mass with isointensity (8 cases) (Figs. 9a and 10) and marginal hyperintensity ( 3 cases) was detected, whereas in 7 cases the signal of the soft tissue mass was heterogeneous. On T2WI, an aggressive soft tissue mass with heterogeneous iso- or hyperintensity (11 cases) (Figs. 9b and 11) was detected.

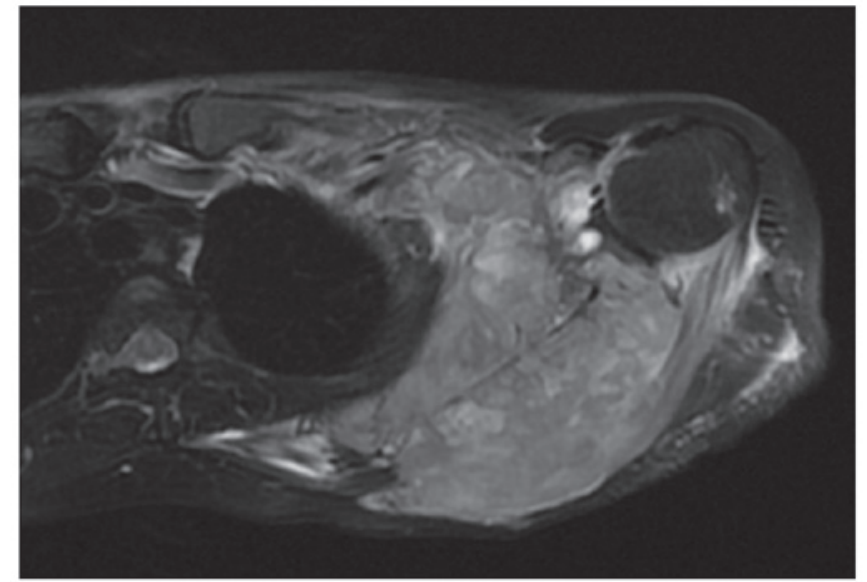

Figure 11. Patient 8. Aggressive soft tissue mass with heterogeneous iso- or hyperintensity detected around the left scapula on T2-weighted images.

On contrast-enhanced T1WI, marked heterogeneous enhancement (Fig. 9c and d) was present in 10 cases and intermediate heterogeneous enhancement in 1 case.

Follow-up. The clinical course of the 15 patients is summarized in Table II. Of the 15 patients, 14 developed no distant metastasis and the remaining patient exhibited lung and hepatic metastasis at the time of diagnosis. Of the 15 patients, 7 underwent surgical treatment, 3 received chemotherapy 


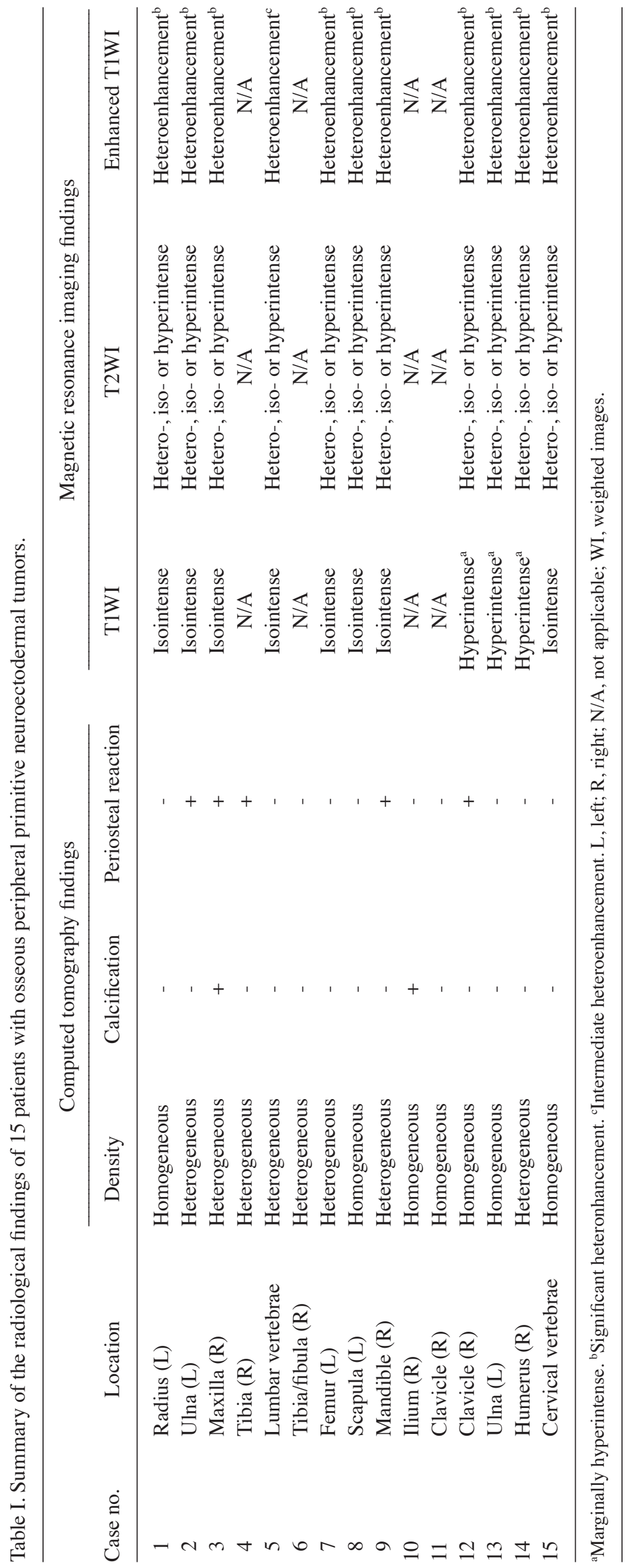


Table II. General data and clinical course of 15 patients with osseous peripheral primitive neuroectodermal tumors.

\begin{tabular}{|c|c|c|c|c|}
\hline $\begin{array}{l}\text { Case no./ age } \\
\text { (years)/ gender }\end{array}$ & Treatment modalities & $\begin{array}{l}\text { Follow-up } \\
\text { (months) }\end{array}$ & $\begin{array}{l}\text { Recurrence/metastasis } \\
\text { during follow-up }\end{array}$ & Status \\
\hline $1 / 38 / \mathrm{F}$ & $\begin{array}{l}\text { Surgical resection, } \\
\text { postoperative chemotherapy }\end{array}$ & 7 & $\begin{array}{l}\text { Local recurrence and } \\
\text { distant metastasis }\end{array}$ & Alive \\
\hline $2 / 22 / \mathrm{F}$ & Chemotherapy & 24 & Remission & Alive \\
\hline $3 / 16 / \mathrm{M}$ & Chemotherapy, radiotherapy & 23 & Remission & Alive \\
\hline $4 / 64 / \mathrm{F}$ & No treatment & 6 & Distant metastasis & Deceased \\
\hline $5 / 19 / \mathrm{M}$ & Radiotherapy, chemotherapy & 5 & $\begin{array}{l}\text { Distant metastasis at the } \\
\text { time of diagnosis }\end{array}$ & Deceased \\
\hline $6 / 25 / \mathrm{M}$ & Chemotherapy & 19 & Remission & Alive \\
\hline 7/17/M & $\begin{array}{l}\text { Preoperative chemotherapy, } \\
\text { surgical resection }\end{array}$ & 23 & $\begin{array}{l}\text { Local recurrence and } \\
\text { distant metastasis }\end{array}$ & Deceased \\
\hline $8 / 54 / \mathrm{F}$ & $\begin{array}{l}\text { Preoperative chemotherapy, } \\
\text { surgical resection }\end{array}$ & 6 & $\begin{array}{l}\text { Local recurrence and } \\
\text { distant metastasis }\end{array}$ & Alive \\
\hline $9 / 16 / \mathrm{M}$ & Chemotherapy, radiotherapy & 18 & Distant metastasis & Deceased \\
\hline $10 / 43 / \mathrm{F}$ & Radiotherapy, chemotherapy & 12 & Distant metastasis & Deceased \\
\hline $11 / 17 / \mathrm{F}$ & $\begin{array}{l}\text { Surgical resection, postoperative } \\
\text { chemotherapy }\end{array}$ & 18 & $\begin{array}{l}\text { Local recurrence and } \\
\text { distant metastasis }\end{array}$ & Alive \\
\hline $12 / 19 / \mathrm{M}$ & $\begin{array}{l}\text { Surgical resection, postoperative } \\
\text { chemotherapy }\end{array}$ & 22 & $\begin{array}{l}\text { Local recurrence and } \\
\text { distant metastasis }\end{array}$ & Alive \\
\hline $13 / 28 / \mathrm{M}$ & $\begin{array}{l}\text { Surgical resection, postoperative } \\
\text { chemotherapy }\end{array}$ & 6 & $\begin{array}{l}\text { No local recurrence and } \\
\text { distant metastasis }\end{array}$ & Alive \\
\hline $14 / 30 / \mathrm{M}$ & Chemotherapy & 8 & Distant metastasis & Deceased \\
\hline $15 / 25 / \mathrm{M}$ & $\begin{array}{l}\text { Surgical resection, postoperative } \\
\text { chemotherapy }\end{array}$ & 14 & $\begin{array}{l}\text { Local recurrence and } \\
\text { distant metastasis }\end{array}$ & Deceased \\
\hline
\end{tabular}

F, female; M, male.

alone and 4 received chemoradiotherapy alone. One patient received no treatment and succumbed to the disease 6 months after hospital discharge. Of the 7 surgical patients, 6 developed local recurrence and distant metastasis subsequent to surgery during the follow-up period, of whom 2 patients eventually succumbed to the disease, whereas the remaining patient did not exhibit local recurrence or metastasis. Of the 7 patients who underwent chemotherapy or chemoradiotherapy alone, 3 achieved a remission during the follow-up period, whereas the remaining 4 patients succumbed to lymph node, pulmonary, hepatic, osseous or meningeal metastasis.

\section{Discussion}

The first case of pPNET, occurring in the ulnar nerve, was reported by Stout in 1918 and the tumor was composed of small round cells, focally arranged as rosettes (4). Ewing reported an undifferentiated, diffuse, small round-cell tumor occurring in the diaphysis of long bones in 1921; that type of tumor was eventually named Ewing's sarcoma (ES) (5). In 1984, Jaffe (6) analyzed the pathological sections of 4 cases that had been diagnosed as bone ES by previous clinical, X-ray and pathological examinations and identifed Homer-Wright rosettes by microscopy and neurospecific enolase (NSE) expression by immunohistochemical staining; thus, these cases were diagnosed as neuroectodermal tumors, rather than ES, and were the first reported cases of osseous pPNET. It was recently demonstrated that pPNET and ES may be further differentiated using characteristics observed on electron microscopic and immunohistochemical examination. For example, neurotubules, neurofilaments and neurosecretory granules may be observed under an electron microscope, whereas O13, CD99, S100-protein, vimentin, chorionic gonadotropin $\alpha(\mathrm{CgA})$ and NSE may be identified by immunohistochemical staining in PNETs (7-12).

The PNETs, a subtype of the family of small round-cell malignancies, are mainly found in the central nervous system (CNS). Rarely, however, PNETs may be found outside the CNS. These pPNETs are most common in the thoracopulmonary region, followed by the abdomen, pelvic cavity and retroperitoneum. Bone is a rare location and, to date, there have been few reports of osseous pPNET in the literature (3). To the best of our knowledge, this is the first English language study exclusively investigating the CT and MRI findings of osseous pPNETs. Osseous pPNETs may occur in any bone in every age group, but mainly affect children and adolescents and exhibit a marginal male predominance. Patients often present with a rapidly enlarging mass and associated compression symptoms $(3,12)$. Consistent with previous reports $(9,11)$, osseous pPNET mainly affected males, adolescents and young adults in the present study (Table II). The 15 patients presented with varying degrees 
of pain and 11 patients presented with local edema and a progressively growing mass. The tumors were located in the limb bones ( 7 cases), vertebrae (2 cases), clavicle (2 cases), scapula (1 case), ilium (1 case), maxilla (1 case) and mandible ( 1 case). It should be noted that pPNETs of the maxilla and mandible are extremely rare. Following a review of the literature, only 16 pPNET cases of the mandible (13) and 13 of the maxilla (14) have been reported.

The CT findings of osseous pPNETs include destructive lesions with a sizeable soft tissue mass and, occasionally, with periosteal reaction $(3,13)$. The soft tissue mass is usually isodense or marginally hypodense compared with normal muscle $(1,3,15)$. When the tumor is smaller, its density is often homogeneous. However, when the tumor is larger, it often exhibits isodensity with patchy hypodense necrotic areas. Tumor calcification is uncommon $(11,12)$. In the present study, 13 of the 15 cases exhibited lytic bone lesions; all 15 cases exhibited surrounding soft tissue mass formation and 5 cases exhibited associated periosteal reaction. In 9 cases the soft tissue mass was heterogeneous, with different sizes of lower-density necrotic areas. Two cases of soft tissue mass exhibited calcification. These findings were consistent with the CT findings of osseous pPNET. Of note, osseous pPNET is a highly invasive malignant tumor and, therefore, it theoretically lacks the time required for calcification or ossification. Thus, the 2 cases exhibiting calcifications may be due to the pressure exerted on normal bone tissue, which may be observed among the CT findings of primary bone lymphoma (formation of sequestra) (16).

On T1WI, the majority of pPNETs are isointense or marginally hyperintense compared with normal muscle, and may contain hyperintense hemorrhagic areas. On T2WI, the majority of pPNETs are heterogeneous iso- or hyperintense. On contrast-enhanced T1WI, the tumor is often homogeneous when it is smaller and heterogeneous when it grows larger and exhibits areas of hemorrhage or necrosis $(8,9)$. In the present study, 11 cases exhibited a surrounding soft tissue mass on MRI. On T1WI, a soft tissue mass exhibiting isointensity ( 8 cases) or marginal hyperintensity ( 3 cases) was identified. On T2WI, a soft tissue mass exhibiting heterogeneous iso- or hyperintensity (11 cases) was identified. On contrast-enhanced T1WI, marked heterogeneous enhancement was present in 10 cases and intermediate heterogeneous enhancement in 1 cases. These findings were consistent with those of previous reports $(3,4)$. MRI is a sensitive method for displaying and accurately evaluating the extent of the lesions, evaluating treatment effectiveness and detecting the presence of distant metastases during the follow-up period. The two cases of the present study and those in other literature reports demonstrated that the ability of MRI to detect change is superior to that of CT, even following chemotherapy (11).

pPNETs are often associated with distant metastases and local recurrence following treatment, as well as poor prognosis. The most common recurrence is characterized by localized soft tissue masses and distant metastases, often occurring in the lung, bone, liver, adrenal gland, brain and retroperitoneum (15). In the present study, 1 patient had pulmonary and hepatic metastases at the time of diagnosis, 6 patients developed local recurrence and distant metastasis following surgery during the follow-up period and 4 patients developed distant metastasis following chemotherapy or chemoradiotherapy. A total of 7 patients succumbed to lymph node, pulmonary, hepatic, osseous or meningeal metastasis during the follow-up period.

In conclusion, osseous pPNETs mainly affect males aged $<30$ years. The patients often present with varying degrees of pain, local edema and a progressively growing mass. The CT findings of osseous pPNET include destructive lesions with a sizeable soft tissue mass and, occasionally, with periosteal reaction. Tumor calcification is uncommon. The MRI findings include aggressive soft tissue mass with isointensity on T1WI and iso- or hyperintensity on T2WI and markedly heterogeneous tumors following enhancement. The CT and MRI findings demonstrated that the tumor originated in the bone marrow cavity and exhibited a bone-centric growth pattern. Although the imaging characteristics of pPNETs may be non-specific, CT and MRI may be useful in delineating the extent of the tumor, identifying distant metastases, predicting resectability and monitoring treatment.

\section{References}

1. Schulman H, Newman-Heinman N, Kurtzbart E, Maor E, Zirkin $\mathrm{H}$ and Laufer L: Thoracoabdominal peripheral primitive neuroectodermal tumors in childhood: radiological features. Eur Radiol 10: 1649-1652, 2000.

2. Gong J, Zhang Y, Zuo M, et al: Imaging findings of abdominal peripheral primitive neuroectodermal tumor: report of four cases with pathological correlation. Clin Imaging 33: 196-199, 2009.

3. Ibarburen C, Haberman JJ and Zerhouni EA: Peripheral primitive neuroectodermal tumors. CT and MRI evaluation. Eur J Radiol 21: 225-232, 1996.

4. Stout AP: A tumor of the ulnar nerve. Proc NY Pathol Soc 12: 2-12, 1918.

5. Ewing J: Diffuse endothelioma of bone. Proc NY Pathol Soc 21: 17-24, 1921.

6. Jaffe R: The neuroectodermal tumor of bone. Am J Surg Pathol 8: 885-898, 1984.

7. Linnoila RI, Tsokos M, Triche TJ, Marangos PJ and Chandra RS: Evidence for neural origin and PAS-positive variants of the malignant small cell tumor of thoracopulmonary region ('Askin tumor'). Am J Surg Pathol 10: 124-133, 1986.

8. Carvajal R and Meyers P: Ewing's sarcoma and primitive neuroectodermal family of tumors. Hematol Oncol Clin North Am 19: 501-525, 2005.

9. de Alava E and Gerald WL: Molecular biology of the Ewing's sarcoma/primitive neuroectodermal tumor family. J Clin Oncol 18: 204-213, 2000.

10. Jones JE and McGill T: Peripheral primitive neuroectodermal tumors of the head and neck. Arch Otolaryngol Head Neck Surg 121: 1392-1395, 1995.

11. Dick EA, McHugh K, Kimber C and Michalski A: Imaging of non-central nervous system primitive neuroectodermal tumours: diagnostic features and correlation with outcome. Clin Radiol 56: 206-215, 2001.

12. Khong PL, Chan GC, Shek TW, Tam PK and Chan FL: Imaging of peripheral PNET: common and uncommon locations. Clin Radiol 57: 272-277, 2002.

13. Yeh $\mathrm{CH}$, Yeow $\mathrm{KM}$, Chu SY, et al: Imaging findings in mandibular primitive neuroectodermal tumour: a report of a rare case and review of the literature. Dentomaxillofac Radiol 40: 451-456, 2011.

14. Hormozi AK, Ghazisaidi MR and Hosseini SN: Unusual presentation of peripheral primitive neuroectodermal tumor of the maxilla. J Craniofac Surg 21: 1761-1763, 2010.

15. Zhang WD, Chen YF, Li CX, Zhang L, Xu ZB and Zhang FJ: Computed tomography and magnetic resonance imaging findings of peripheral primitive neuroectodermal tumors of the head and neck. Eur J Radiol 80: 607-611, 2011.

16. Mulligan ME and Kransdorf MJ: Sequestra in primary lymphoma of bone: prevalence and radiologic features. AJR Am J Roentgenol 160: 1245-1248, 1993. 